\title{
Evaluation of Acoustic Radiation Force Impulse Imaging (ARFI) for the Determination of Liver Stiffness Using Transient Elastography as a Reference in Children
}

Authors

Affiliations
A. Sagir ${ }^{1}$, D. Ney ${ }^{2,4}$, J. Oh ${ }^{3,4}$, S. Pandey ${ }^{4}$, G. Kircheis ${ }^{1}$, E. Mayatepek ${ }^{4}$, D. Häussinger

Affiliation addresses are listed at the end of the article
Key words

- abdomen

- diagnostic radiology

- safety

\begin{abstract}
$\nabla$

Purpose: Transient elastography (Fibroscan $^{\complement}$; (FS)) and acoustic radiation force impulse imaging (ARFI) represent noninvasive, user-friendly and quick methods providing an objective and reproducible measure of liver stiffness. The aim of the study was to evaluate cut-off values and performance of ARFI measurements in children using transient elastography as a reference.

Methods/Patients: A total of 198 children were enrolled in this study. All patients underwent liver stiffness measurements with FS (FS-LS) as well as ARFI (with shear wave velocity quantification; ARFI-SWV) and the performance of ARFI in comparison to FS was studied.
\end{abstract}

\section{Background}

Bibliography DOI http://dx.doi.org/ 10.1055/s-0035-1554659 Ultrasound International Open 2015; 1: E2-E7 (c) Georg Thieme Verlag KG Stuttgart · New York ISSN 2199-7152

\section{Correspondence}

Dr. Abdurrahman Sagir Klinik für Gastroenterologie, Hepatologie und Infektiologie Uniklinik Düsseldorf Moorenstraße 5 40231 Düsseldorf Germany Tel.: + 49/211/8118 648 Fax: $+49 / 211 / 8104041$ sagirabdu@hotmail.com

\section{License terms}

\section{(ब)(1) $\Theta \circledast$}

$\nabla$

Liver biopsy is currently considered the gold standard for the precise assessment of hepatic fibrosis [1]. However, it is an invasive procedure with rare but severe complications. In addition, the accuracy of liver biopsy for the assessment of fibrosis may suffer from interobserver variability [2-4]. Therefore, research has recently been focused on the evaluation of noninvasive methods for the assessment of liver fibrosis, such as routine biological and hematologic tests, surrogate serum fibrosis markers and measurement of liver elasticity [5-7]. Transient elastography (FibroScan $^{\odot}(\mathrm{FS})$ ) is an established rapid, noninvasive, and reproducible method for measuring liver stiffness (FS-LS). A strong association of FS-LS and the degree of liver fibrosis has already been demonstrated in adult patients with chronic hepatitis [8,9]. A cut-off value of $13 \mathrm{kPa}$ has been defined for the discrimination between liver fibrosis and cirrhosis [8]. The cut-off value of $7.6 \mathrm{kPa}$ has been suggested for the discrimination
Results: Significantly higher rates of successful measurements were found for ARFI compared to FS (198/198 (100\%) vs. 160/198 (80.8\%); p<0.001). ARFI-SWV correlated significantly with FS-LS $(r=0.751, p=0.001)$. ARFI-SWV increased significantly with the stage of fibrosis $(1.19+0.15 \mathrm{~m} / \mathrm{s}$ for patients with FS-LS $<7.6 \mathrm{kPa}) ; 1.34+0.22 \mathrm{~m} / \mathrm{s}$ for patients with $7.6<\mathrm{FS}-\mathrm{LS}<13.0 \mathrm{kPa}$ ); and $1.83+0.58 \mathrm{~m} / \mathrm{s}$ for patients with FS-LS> $13.0 \mathrm{kPa}$ ). ARFI-SWV cut-off values were identified for no significant fibrosis $(1.31 \mathrm{~m} / \mathrm{s}$; sensitivity $61.8 \%$ and specificity $79.5 \%$ ) and for liver cirrhosis $(1.63 \mathrm{~m} / \mathrm{s}$; sensitivity $70.0 \%$ and specificity $97.4 \%$ ). The median values of liver stiffness measured by FS were age-dependent in 90 children without liver diseases with 4.8, 5.6, and $5.7 \mathrm{kPa}$ in children $0-5$, $6-11$, and $12-18$ years, respectively.

of fibrosis stage from no significant fibrosis [10]. The limitation of FS has been described in several studies $[11,12]$. There is considerable experience for FS for adults, but experience is limited for children [13].

Another noninvasive tool for the detection of liver fibrosis is the acoustic radiation force impulse (ARFI) imaging technology [14]. ARFI imaging has been incorporated into a conventional ultrasound (US) device (Acuson S2000; Siemens Medical Solutions, Mountain View, Calif). ARFI imaging technology involves the mechanical excitation of tissue using short-duration acoustic pulses in a region of interest, producing shear waves that spread away from the region of interest $[15,16]$. By recording the shear wave front and correlating these measurements with the elapsed time, the shear wave velocity $(\mathrm{SWV})(\mathrm{m} / \mathrm{s})$ can be quantified (ARFI-SWV). The SWV increases with the stiffness. Thus, the measured SWV is an intrinsic and reproducible property of the tissue $[17,18]$. A significant correlation between ARFI imaging, serum fibrosis marker 
tests, and the histological fibrosis stage was reported in some studies [19-21]. In this study we compare FS-LS with ARFI-SWV in children. The aim of the study was to evaluate cut-off values and performance of ARFI measurements in children by using transient elastography as a reference.

Finally, the age dependence of FS-LS and ARFI-SWV should be investigated.

\section{Methods \\ $\nabla$}

\section{Patients}

This study was approved by the ethics committee of Heinrich Heine University. Informed consent was obtained from all participating subjects. A total of 198 patients who had consulted the University Children's Hospital, Department of General Pediatrics, Neonatology, and Pediatric Cardiology Düsseldorf, Germany were included in this study. Patients with aminotransferases higher than 5 times the normal upper limit were not included in the study. The etiology of the liver disease was determined according to standard diagnostic criteria. The patient characteristics are shown in $\bullet$ Table 1.

\section{Liver stiffness measured by FS}

Liver stiffness was measured in fasting patients. Details of the technical background and examination procedure have been described in detail [22]. The tip of the probe was placed on the skin between the ribs over the right liver lobe. The small probe (s-probe) was used for children with a thoracic diameter less than $45 \mathrm{~cm}$. All other children were examined with the medium probe (m-probe). The measurement depth was between 15 and $40 \mathrm{~mm}$ below the skin surface for the s-probe and 25 and $65 \mathrm{~mm}$ for the m-probe. 10 measurements were performed in each patient. Determination of the liver stiffness was considered valid when a success rate of at least $60 \%$ was obtained and an interquartile range (IQR) of less than $30 \%$ was reached. The results were expressed in kilopascal $(\mathrm{kPa})$. The median value was taken as representative.

Table 1 Characteristics of patients at the time of liver stiffness measurement ( $n=198)$.

\begin{tabular}{|ll}
\hline Patients, $\mathrm{n}$ & 198 \\
\hline Male, $\mathrm{n}(\%)$ & $116(58.5 \%)$ \\
\hline Age (years) & $8 . \pm 5.2$ (range 6 weeks - 18 years) \\
\hline ALT (IU/I) & $22.5 \pm 13.6$ (range 5-84) \\
\hline AST (IU/I) & $33.1 \pm 13.3$ (range 10-86) \\
\hline GGT (IU/I) & $21.4 \pm 23.3$ (range 3-95) \\
\hline Total bilirubin (mg/dl) & $0.46 \pm 0.57$ (range 0.1-4.4) \\
\hline Length (cm) & $130.4 \pm 35.3$ \\
\hline Weight (kg) & $35.7 \pm 21.9$ \\
\hline BMI (kg/m²) & $18.4 \pm 4.1$ (range 12.1-37.7) \\
\hline Skin-liver capsule distance (cm) & $1.77 \pm 0.43$ \\
\hline Chronic liver diseases, $\mathrm{n}$ (\%) & $18(9 \%)$ \\
\hline Metabolic diseases with hepatic & $39(20 \%)$ \\
\hline involvement, $\mathrm{n}(\%)$ & $12(6 \%)$ \\
\hline Heart diseases, $\mathrm{n}(\%)$ & $90(45 \%)$ \\
\hline $\begin{array}{l}\text { Diseases without hepatic } \\
\text { involvement, } \mathrm{n}(\%)\end{array}$ & $39(20 \%)$ \\
\hline Others, $\mathrm{n}$ (\%) & \\
\hline
\end{tabular}

\section{ARFI-SWV determination}

In all patients, ARFI imaging (Acuson S2000, Virtual Touch Tissue Quantification mode) and transient elastography (TE; FibroScan; Echosens, Paris, France) were performed on the same day. The examinations were performed in the right lobe of the liver with the $6 \mathrm{C} 1 \mathrm{HD}$ probe, through the intercostal space, at the same site as the transient elastography measurement. A measurement depth of $2 \mathrm{~cm}$ below the liver capsule was chosen to standardize the examination for ARFI-SWV. 10 measurements were performed in each patient. Determination of the liver stiffness was considered valid when a success rate of at least $60 \%$ was obtained and an interquartile range (IQR) of less than $30 \%$ was reached. The mean value of 10 measurements was taken as representative.

\section{Statistical analysis}

Data were entered in SPSS (version 21.0, Inc., Munich, Germany). $\mathrm{A} \mathrm{X}^{2}$ or Fisher's exact test (F-test) was used for the comparison of categorical variables, and a Mann-Whitney test was used for the comparison of continuous variables. The significance level was set to 0.05 , and all p-values were 2-tailed. A Pearson's test was performed to study the correlation between FS-LS and ARFISWV.

For no significant fibrosis (FS $\leq 7.6 \mathrm{kPa}$ ) and liver cirrhosis (FS $>13.0 \mathrm{kPa}$ ), the diagnostic performance of ARFI was assessed using receiver operating characteristic (ROC) curves. The ROC curve is a plot of sensitivity vs. 1 - specificity for all possible cutoff values. The most commonly used index of accuracy is the area under the ROC curve (AUROC). AUROC values close to 1.0 indicate high diagnostic accuracy. ROC curves were generated for patients with $\mathrm{FS} \leq 7.6 \mathrm{kPa}$ and patients with $\mathrm{FS}>13 \mathrm{kPa}$. Optimal cut-off values for ARFI were chosen to maximize the sum of sensitivity and specificity, and positive and negative predictive values were computed for these cut-off values. Using this analysis, SWV cut-off values were identified for patients with no significant fibrosis ( $\mathrm{FS} \leq 7.6 \mathrm{kPa}$ ) and patients with liver cirrhosis (FS $>13.0 \mathrm{kPa}$ ). All patients with valid liver stiffness measurement by FS and ARFI were included in these analysis.

\section{Results}

\section{$\nabla$}

A total of 198 pediatric patients were enrolled in this study. FS-LS ranged from 2.3 to $75.0 \mathrm{kPa}$ (median $5.4 \mathrm{kPa}$ ) and ARFISWV ranged from 0.82 to $2.96 \mathrm{~m} / \mathrm{s}$ (mean $1.25 \pm 0.3 \mathrm{~m} / \mathrm{s}$ ). The mean depth of the area where ARFI-SWV measurements were performed was $3.77 \pm 0.45 \mathrm{~cm}$. The overall success rate was $92.5 \pm 11.9 \%$ for FS compared to $96.7 \pm 7.6 \%$ for ARFI $(p<0.001)$. 146 patients $(73.7 \%)$ were examined with the s-probe (infants and younger children) and 52 patients (26.3\%) with the m-probe (older children).

A valid liver stiffness determination (success rate of at least 60\%) and IQR $<30 \%$ was observed in $160 / 198(80.8 \%)$ by FS compared to $198 / 198(100 \%)$ by ARFI ( $\mathrm{p}<0.001)$.

After the exclusion of all patients with an invalid liver stiffness determination by FS (success rates below $60 \%$ or ICR $>30 \%$ ), 160 patients still remained for the following analysis ( $\bullet$ Fig. 1). Of the remaining 160 children, 70 had known chronic liver disease or metabolic diseases with hepatic involvement and 90 children had a healthy liver. 


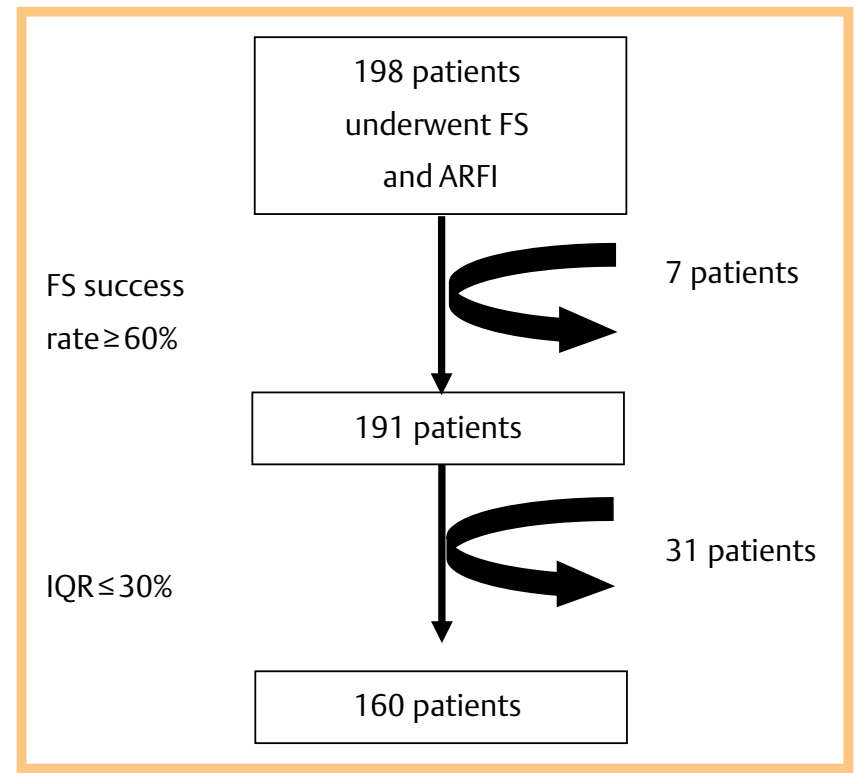

Fig. 1 Selection process for valid measurement of liver stiffness by transient elastography.

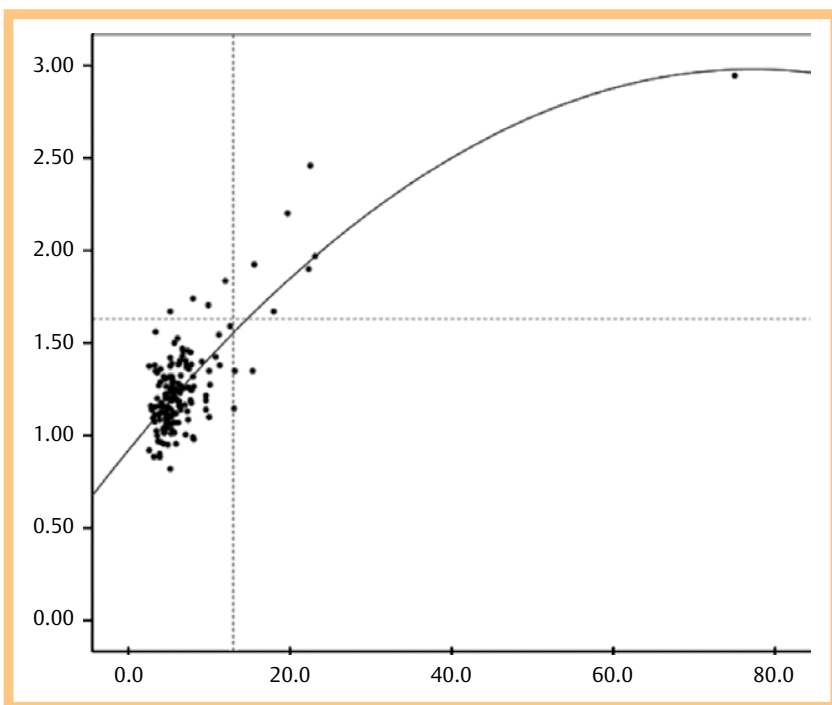

Fig. 2 Correlation of liver stiffness measured by FS and ARFI $(r=0.751$; $p=0.001$ ); the vertical line represents the cut-off value of $13 \mathrm{kPa}$ for $F S$ and the horizontal line represents the cut-off value $1.63 \mathrm{~m} / \mathrm{s}$ for ARFI ( $n=160$ ); by using the cut-off value of 1.63 for the discrimination of liver fibrosis from liver cirrhosis for ARFI-SWV, 153 of the 160 patients (95.6\%) were classified correctly. $3(1.9 \%)$ patients were classified false-negative (FS-LS $>13 \mathrm{kPa}$ and ARFI-SWV $<1.63 \mathrm{~m} / \mathrm{s}$ ), and $4(2.5 \%$ ) were classified falsepositive (FS-LS $<13 \mathrm{kPa}$ and ARFI-SWV $>1.63 \mathrm{~m} / \mathrm{s}$.

\section{Correlation of ARFI with FS}

A Pearson test was performed to analyze the correlation between FS-LS and ARFI-SWV. There was a significant correlation between these 2 methods ( $<<0.001 ; r=0.751$; $\odot$ Fig. 2 ).

In consideration of the cut-off values for the different stages of liver fibrosis detected by FS, the following frequencies were observed: $130(81.3 \%)$ children did not show significant fibrosis (FS-LS $\leq 7.6 \mathrm{kPa}), 22(13.7 \%)$ showed significant fibrosis $(7.6 \mathrm{kPa}<$ FS-LS $\leq 13.0 \mathrm{kPa}$ ), and $8(5.0 \%$ ) showed cirrhosis (FS-LS $>13.0 \mathrm{kPa}$ ). The mean ARFI-SWV was $1.19 \pm 0.15 \mathrm{~m} / \mathrm{s}$ (range $0.82-1.67 \mathrm{~m} / \mathrm{s}$ ) for patients with no significant fibrosis (FS-LS $<7.6 \mathrm{kPa}$ ), compared to $1.34 \pm 0.22 \mathrm{~m} / \mathrm{s}$ (range $0.98-1.84 \mathrm{~m} / \mathrm{s}$ ) for patients with

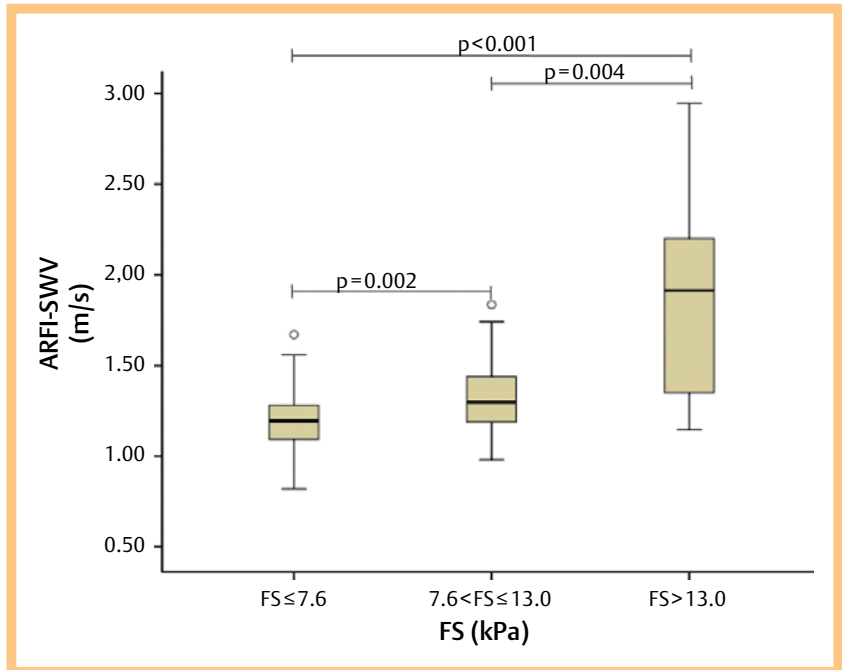

Fig. 3 Acoustic radiation force impulse imaging shear wave velocity (ARFI-SWV) for the different stages of fibrosis (FS<7.6 kPa: no significant fibrosis; $7.6 \mathrm{kPa}<\mathrm{FS}<13.0 \mathrm{kPa}$ : significant fibrosis; FS>13.0 kPa: liver cirrhosis. Box plots show median values with $25^{\text {th }}$ and $75^{\text {th }}$ percentiles of shear wave velocity determined by ARFI.

significant liver fibrosis, and $1.83 \pm 0.58 \mathrm{~m} / \mathrm{s}$ (range $1.15-2.95 \mathrm{~m} / \mathrm{s}$ ) for patients with liver cirrhosis. ARFI-SWV was significantly different between patients according to their fibrosis stage with overlapping confidence intervals. O Fig. 3 shows box plots of ARFI-SWV for the 3 groups.

Receiver operating characteristic (ROC) analysis of ARFI - Fig. 4a, b show the diagnostic value (ROC curves) of the liver stiffness measurement for patients with FS-LS $<7.6 \mathrm{kPa}(\boldsymbol{O}$ Fig. 4a) and patients with FS-LS>13.0 kPa ( $\bullet$ Fig. $4 \mathbf{b}$ ). Corresponding AUROC values and $95 \%$ confidence intervals were 0.785 (95\% CI: $0.659-0.857$ ) for $\mathrm{FS}-\mathrm{LS}<7.6 \mathrm{kPa}$ and 0.890 (95\% CI: 0.724-1.00) for FS-LS $>13 \mathrm{kPa}$. Based on the ROC curves, the optimal cut-off values for ARFI were chosen to maximize the sum of sensitivity and specificity. These cut-off levels were $1.31 \mathrm{~m} / \mathrm{s}$ (sensitivity $61.8 \%$ and specificity $79.5 \%$ for FS-LS $<7.6 \mathrm{kPa}$ ) and $1.63 \mathrm{~m} / \mathrm{s}$ (sensitivity $70.0 \%$ and specificity $97.4 \%$ for $\mathrm{FS}-\mathrm{LS}>13 \mathrm{kPa}$ ) (० Table 2).

Using these cut-off values for ARFI-SWV, 153 of the 160 patients (95.6\%) were classified correctly. $3(1.9 \%)$ patients were falsenegative (FS-LS $\geq 13 \mathrm{kPa}$ and ARFI-SWV $<1.63 \mathrm{~m} / \mathrm{s}$ ), and $4(2.5 \%)$ were false-positive (FS-LS $<13 \mathrm{kPa}$ and ARFI-SWV $\geq 1.63 \mathrm{~m} / \mathrm{s}$ ). Using the cut-off value of $1.63 \mathrm{~m} / \mathrm{s}$, patients with an $\mathrm{FS}>13 \mathrm{kPa}$ were detected with a positive predictive value of $50 \%$ and a negative predictive value of $97.4 \%$.

\section{Age-dependent normal values for TE and ARFI}

In order to analyze age-dependent normal values for TE and ARFI, all patients with diseases with hepatic involvement were excluded (chronic hepatitis B or C $(n=5)$, Wilson's disease $(n=2)$, autosomal recessive polycystic kidney disease $(n=6)$, diabetes mellitus $(n=26)$, non-alcoholic steatohepatitis $(n=16)$, heart dysfunction $(n=4)$, gluconeogenesis $(n=2)$, cystic fibrosis $(n=1)$, tyrosinemia $(n=1)$, celiac sprue $(n=1)$, suspicion of primary sclerosing cholangitis $(n=3)$, ornithine transcarbamylase deficiency $(n=1)$, and post-LTX $(n=1)$ or Schwachman-Diamond syndrome $(n=1)$ ). Of the 160 patients who fulfilled the criteria for a valid measurement by FS, 90 patients remained for the fol- 
lowing analysis. Characteristics of these patients are shown in $\odot$ Table 3.8 children presented a liver stiffness measured by FS $>7.6 \mathrm{kPa}$ (phlegmon $(n=1)$, cervical lymphadenitis $(\mathrm{n}=1)$, allergic reaction $(n=2)$, hematuria $(n=1)$, asthma bronchiale $(n=1)$, depressive disorder $(n=1)$, and arthritis $(n=1))$. The liver stiffness measured by FS correlated significantly with age $(p=0.011 ; r=0.266)$. A significant correlation between ARFI and

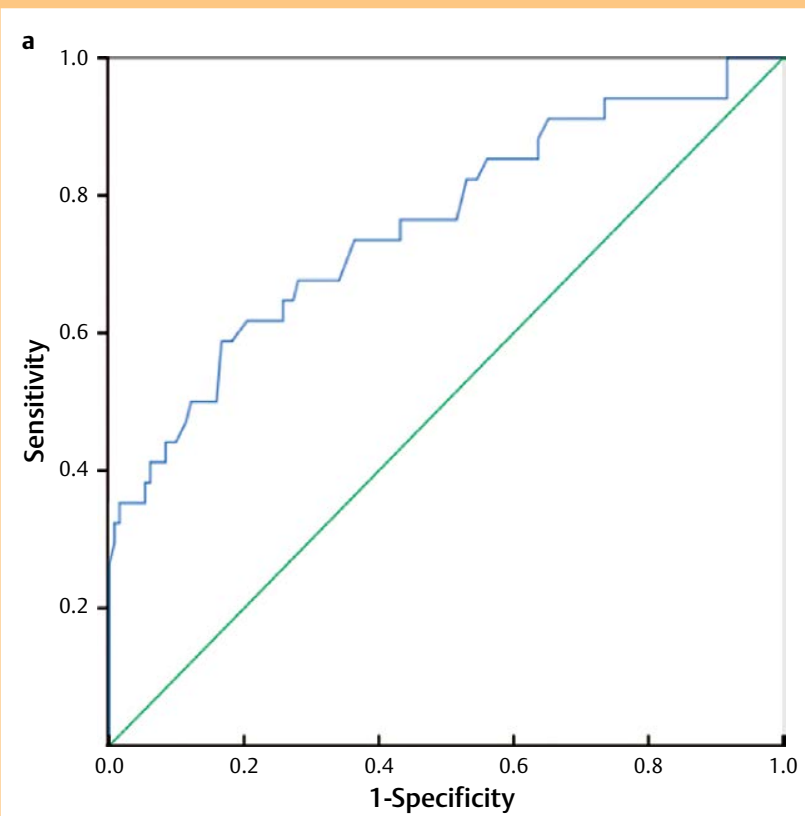

b

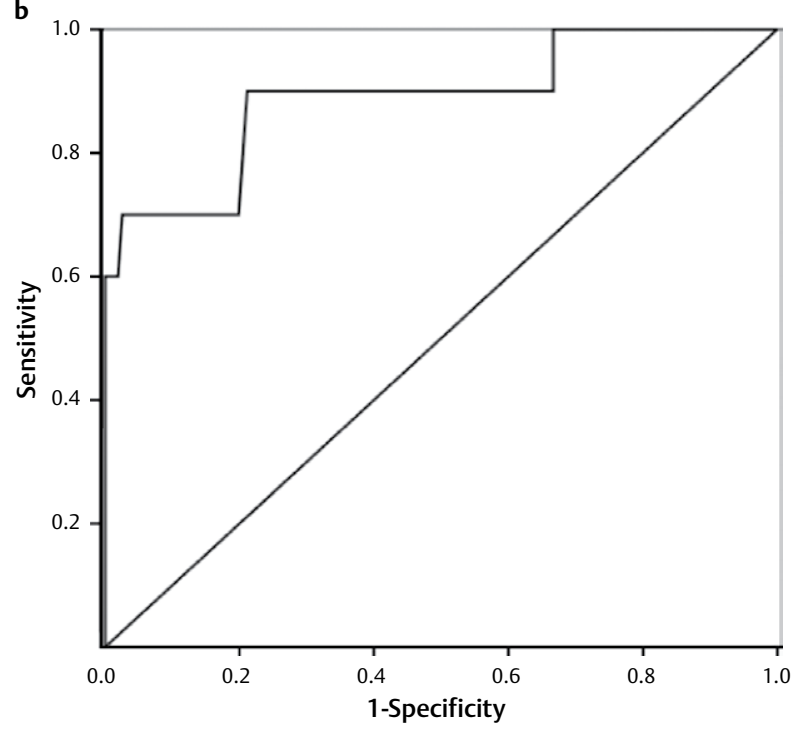

Fig. 4 a Receiver operator characteristic (ROC) curve for ARFI for the prediction of no significant fibrosis ( $\mathrm{FS}<7.6 \mathrm{kPa} ; \mathrm{n}=160$; $95 \% \mathrm{Cl}$ : 0.659 0.0857). b Receiver operator characteristic (ROC) curve for ARFI for the prediction of liver cirrhosis (FS>13.0 kPa; $n=160 ; 95 \% \mathrm{Cl}: 0.724-1.00$ ). age could not be observed $(\mathrm{p}=0.125)$. To study the age dependence of FS, these patients were divided into 3 groups (group1: $0-5$ years $(n=29)$, group $2: 6-11$ years $(n=34)$, and group 3 : $12-18$ years $(n=27)$ ). There was a significant age-dependent difference of the liver stiffness measured by FS with an increase of the values with age: group $1(4.8+1.4 \mathrm{kPa})$, group $2(5.4 \pm 1.3 \mathrm{kPa})$, and group $3(5.5 \pm 1.7 \mathrm{kPa})(\mathrm{p}=0.028$ for group 1 vs. group 2; $\mathrm{p}=0.047$ for group 1 vs. group 3; group 2 vs. group 3 was not significant $\mathrm{p}=0.87$; $\odot$ Table 3 ). The age-specific upper limit of the normal value for FS was determined as the median plus 1.64 times the standard deviation were 7.1, 7.5, and $8.1 \mathrm{kPa}$. An age dependence could not be observed for ARFI $(p=0.56$ for group 1 vs. group $2 ; \mathrm{p}=0.08$ for group 1 vs. group $3 ; \mathrm{p}=0.06$ group 2 vs. group 3).

\section{Discussion}

\section{$\nabla$}

Noninvasive detection of liver fibrosis/cirrhosis has recently become of great interest. Particularly in children, these tools might be able to reduce the numbers of biopsies leading to complications. Data of this study suggest that a noninvasive fibrosis/ cirrhosis assessment by ARFI-SWV represents a good correlation with FS-LS measurement and can be successfully used in pediatric patients in whom FS-LS measurements were not successful. A significantly higher success rate was observed for ARFI compared to FS (100 vs. $80.8 \%$; $p<0.001$ ). In contrast to FS, ARFI is performed under the control of conventional B-mode ultrasound. The observer can select and place the region of interest under visual control. Another advantage of ARFI is that the measuring depth can be varied. We chose a depth of $2 \mathrm{~cm}$ under the liver capsule to standardize the examination.

The mean ARFI-SWV increased significantly with the stage of fibrosis. This result can be underlined by Hanquinet et al. [23]. In their study, they compared ARFI with liver biopsy results and also found an increase of ARFI-SWV with the stage of fibrosis. We defined cut-off values for patients with no-significant fibrosis and patients with liver cirrhosis by using the known cut-off values for FS. They were chosen so that the sum between sensitivity and specificity was maximal. A cut-off value for ARFI-SWV of $1.31 \mathrm{~m} / \mathrm{s}$ was associated with a sensitivity of $61.8 \%$ and a specificity of $79.5 \%$ for patients with FS-LS $<7.6 \mathrm{kPa}$ and a cut-off value of $1.63 \mathrm{~m} / \mathrm{s}$ for patients with FS-LS $>13.0 \mathrm{kPa}$ with a sensitivity of $70.0 \%$ and specificity of $97.4 \%$. The cut-off value for liver cirrhosis indicates high diagnostic accuracy. The cut-off value of $1.63 \mathrm{~m} / \mathrm{s}$ is associated with a positive predictive value of $50 \%$ and a negative predictive value of $97.4 \%$ for the diagnosis of liver cirrhosis.

Furthermore, we excluded all patients with liver-associated disease. Using this population, we defined normal values for healthy children and found a significant age correlation of increased liver stiffness measured by FS. The difference could not be observed by ARFI and may be due to the small range of the values meas-

\begin{tabular}{llllll} 
& AUROC & $\begin{array}{l}\text { Cut-off (m/s) } \\
\text { (ARF-SWV) }\end{array}$ & Sensitivity (\%) & Specificity (\%) & 95\% CI \\
\hline FS $<7.6 \mathrm{kPa}$ & 0.785 & 1.31 & 61.8 & 79.5 & $0.659-0.857$ \\
\hline FS $>13.0 \mathrm{kPa}$ & 0.994 & 1.63 & 70.0 & 97.4 & $0.724-1.00$ \\
\hline \multicolumn{7}{l}{ AUROC: area under the receiver operating characteristic curves; ARFI-SWV: acoustic radiation force impulse imaging shear wa }
\end{tabular}

AUROC: area under the receiver operating characteristic curves; ARFI-SWV: acoustic radiation force impulse imaging shear wave velocity 
Table 3 Characteristics of 90 patients with healthy liver after exclusion of all patients with an invalid liver stiffness measurement by FS and diseases with liver involvement. There was a significant age-dependent difference of the liver stiffness measured by FS with an increase of the values with age.

\begin{tabular}{|c|c|}
\hline Patients, n & 90 \\
\hline Male, n (\%) & 57 (59.4\%) \\
\hline Age (years) & $8.2 \pm 4.9$ \\
\hline ALT (IU/I) & $15.2 \pm 4.4$ (range $9-32$ ) \\
\hline AST (IU/I) & $29.6 \pm 6.5$ (range $10-43$ ) \\
\hline GGT (IU/I) & $12.9 \pm 3.4$ (range $6-28$ ) \\
\hline Total bilirubin (mg/dl) & $0.3 \pm 0.2$ (range $0.1-0.9$ ) \\
\hline BMI $\left(\mathrm{kg} / \mathrm{m}^{2}\right)$ & $18.2 \pm 3.6$ (range $12.1-29.9$ ) \\
\hline \multicolumn{2}{|l|}{ FS-LS $($ mean $\pm S D)$} \\
\hline $0-5$ years $(n=29)$ & $4.8 \pm 1.4 \mathrm{kPa}$ \\
\hline $6-11$ years $(n=34)$ & $5.6 \pm 1.3 \mathrm{kPa}^{*}$ \\
\hline $12-18$ years $(n=27)$ & $5.7 \pm 1.7 \mathrm{kPa}^{* *}$ \\
\hline \multicolumn{2}{|l|}{ ARFI-SWV (mean $\pm S D)$} \\
\hline $0-5$ years $(n=29)$ & $1.20 \pm 0.17 \mathrm{~m} / \mathrm{s}$ \\
\hline $6-11$ years $(n=34)$ & $1.22 \pm 0.15 \mathrm{~m} / \mathrm{s}$ \\
\hline $12-18$ years $(n=27)$ & $1.14 \pm 0.13 \mathrm{~m} / \mathrm{s}$ \\
\hline
\end{tabular}

ured by ARFI. While FS-LS ranged from 3 to $10 \mathrm{kPa}$ in this population, ARFI-SWV ranged from 0.82 to $1.67 \mathrm{~m} / \mathrm{s}$. The age dependence of AFRI-SWV or FS has been shown in some studies, but other studies have not been able to reproduce these results. One reason for this discrepancy could be that ARFI-SWV and FS verify by using different probes. The dependence of the probe being used could be shown for FS in a large study with 527 children (comparison S1 vs. S2 probe) and in another study with the comparison of the M probe with the S2 probe $[24,25]$. In the study of Fontanilla et al., children underwent liver stiffness measurement by ARFI with 2 probes (4C1- and 9L4-transducer) [26]. There was a trend to higher ARFI-SWV by using the 4C1 transducer $(1.19 \pm 0.04 \mathrm{~m} / \mathrm{s})$ compared to the 9L4 transducer $(1.15 \pm 0.04 \mathrm{~m} / \mathrm{s})$, but this difference was not significant.

Another reason might be the different numbers of patients in the studies. A dependence of ARFI on age was observed in studies with a large study cohort, but not in studies with a small cohort [26-29]. The number of examined children seems to influence this observation, and may be due to an insufficient statistical power in small groups.

The published FS and ARFI-SWV studies in children are limited by the fact that only a minority of the children underwent liver biopsy. However, the cut-off values for adults seem to be transferable. None of our patients underwent liver biopsy. Our study includes not only patients with different liver diseases but also healthy individuals without liver diseases. The age-specific upper limit of normal FS for children increased with age. Liver stiffness measured by FS increased in the 3 groups with a significant difference between these groups ( $\bullet$ Table 3 ). If we compare our results with the published data from Engelmann et al., a trend to higher than normal FS values can be detected in all groups [30]. The higher values result from the higher median values and an obvious standard deviation, but the trend to higher values with increasing age is comparable.

Normal values for FS are published for healthy adults and do not differ from the data published by Engelmann et al. and also not from our data. Keeping this in mind, the used cut-off values for liver fibrosis/cirrhosis for adults seem to be transferable to children. Based on these FS cut-off values, we calculated ARFI cut-off values. Our cut-off values for no significant fibrosis of $1.31 \mathrm{~m} / \mathrm{s}$ is exactly the value that was calculated by Noruegas et. al [31]. In this study they used liver biopsy as a reference. The fact that our cut-off value for ARFI does not differ from the cut-off value that was calculated using liver biopsy supports the assumption of the transferability of the FS cut-off values.

In our study, we used the s-probe as well as the m-probe depending on the thoracic diameter. Infants at an age of a few weeks can also be precisely examined using the s-probe. This limitation seems not to apply for ARFI. ARFI is independent from the intercostal wideness and the depth of measurement can be adapted to the distance from the skin surface and liver capsule. This may be the reason why the liver stiffness could be detected in a significantly higher proportion of patients by ARFI than by FS. To our knowledge, this is the first study detecting liver fibrosis/ cirrhosis in children using FS and ARFI with a large study cohort. If we compare our results with the published data, confounding factors such as probe choice, sedation, or food intake have to be taken into account [24]. These factors make the comparison between the studies difficult.

In conclusion, ARFI and FS are 2 noninvasive tools to detect liver fibrosis/cirrhosis. A valid liver stiffness measurement could be performed in a significantly higher proportion by ARFI. Both methods showed a statistically significant correlation. In healthy children, a significant age-related increase of liver stiffness was observed by using FS.

\section{Affiliations}

${ }^{1}$ Klinik für Gastroenterologie, Hepatologie und Infektiologie, Uniklinik Düsseldorf, Düsseldorf, Germany

${ }^{2}$ Kinderklinik, Kath. Kinderkrankenhaus Wilhelmstift, Hamburg, Germany

${ }^{3}$ Klinik für Allgemeine Pädiatrie, Universität Hamburg/Eppendorf, Hamburg, Germany

${ }^{4}$ Klinik für Allgemeine Pädiatrie, Neonatologie und KInderkardiologie,

Uniklinik Düseldorf, Düsseldorf, Germany

\section{References}

1 Bravo AA, Sheth SG, Chopra S. Liver biopsy. N Engl J Med 2001; 344: 495-500

2 Abdi W, Millan JC, Mezey E. Sampling variability on percutaneous liver biopsy. Arch Intern Med 1979; 139: 667-669

3 Bedossa P, Dargere D, Paradis V. Sampling variability of liver fibrosis in chronic hepatitis C. Hepatology 2003; 38: 1449-1457

4 Regev A, Berho M, Jeffers $L J$ et al. Sampling error and intraobserver variation in liver biopsy in patients with chronic HCV infection. Am J Gastroenterol 2002; 97: 2614-2618

5 Anastasiou J, Alisa A, Virtue S et al. Noninvasive markers of fibrosis and inflammation in clinical practice: prospective comparison with liver biopsy. Eur J Gastroenterol Hepatol 2009

6 Oudry J, Chen J, Glaser KJ et al. Cross-validation of magnetic resonance elastography and ultrasound-based transient elastography: a preliminary phantom study. J Magn Reson Imaging 2009; 30: 1145-1150

7 Huwart L, van Beers BE. MR elastography. Gastroenterol Clin Biol 2008; 32: $68-72$

8 Erhardt A, Lorke J, Vogt $C$ et al. Transient elastography for diagnosing liver cirrhosis. Dtsch Med Wochenschr 2006; 131: 2765-2769

9 Castera L, Vergniol J, Foucher J et al. Prospective comparison of transient elastography, Fibrotest, APRI, and liver biopsy for the assessment of fibrosis in chronic hepatitis C. Gastroenterology 2005; 128: 343-350

10 Friedrich-Rust M, Ong MF, Martens $S$ et al. Performance of transient elastography for the staging of liver fibrosis: a meta-analysis. Gastroenterology 2008; 134: 960-974

11 Sagir A, Erhardt A, Schmitt $M$ et al. Transient elastography is unreliable for detection of cirrhosis in patients with acute liver damage. Hepatology 2008; 47: 592-595

12 Foucher J, Castera L, Bernard PH et al. Prevalence and factors associated with failure of liver stiffness measurement using FibroScan in a prospective study of 2114 examinations. Eur J Gastroenterol Hepatol 2006; 18: 411-412 
13 Tsochatzis EA, Gurusamy KS, Ntaoula S et al. Elastography for the diagnosis of severity of fibrosis in chronic liver disease: a meta-analysis of diagnostic accuracy. J Hepatol 2011; 54: 650-659

14 Nightingale K, McAleavey S, Trahey G. Shear-wave generation using acoustic radiation force: in vivo and ex vivo results. Ultrasound Med Biol 2003; 29: 1715-1723

15 Frizzell LA, Carstensen EL. Shear properties of mammalian tissues at low megahertz frequencies. J Acoust Soc Am 1976; 60: 1409-1411

16 Palmeri ML, Frinkley KD, Zhai L et al. Acoustic radiation force impulse (ARFI) imaging of the gastrointestinal tract. Ultrason Imaging 2005; 27: 75-88

17 Zhai L, Palmeri ML, Bouchard RR et al. An integrated indenter-ARFI imaging system for tissue stiffness quantification. Ultrason Imaging 2008; 30: 95-111

18 Mauldin FW Jr, Zhu HT, Behler RH et al. Robust principal component analysis and clustering methods for automated classification of tissue response to ARFI excitation. Ultrasound Med Biol 2008; 34: 309-325

19 Bota S, Herkner H, Sporea I et al. ARFI elastography versus transient elastography for the evaluation of liver fibrosis. Liver Int 2013; 33: 1138-1147

20 Nierhoff J, Chávez Ortiz AA, Herrmann E et al. The efficiency of acoustic radiation force impulse imaging for the staging of liver fibrosis: a meta-analysis. Eur Radiol 2013; 23: 3040-3053

21 Sporea I, Bota S, Peck-Radosavljevic $M$ et al. Acoustic Radiation Force Impulse elastography for fibrosis evaluation in patients with chronic hepatitis C: an international multicenter study. Eur J Radiol 2012; 81: 4112-4118

22 Sandrin L, Fourquet B, Hasquenoph JM et al. Transient elastography: a new noninvasive method for assessment of hepatic fibrosis. Ultrasound Med Biol 2003; 29: 1705-1713
23 Hanquinet S, Rougemont AL, Courvoisier D et al. Acoustic radiation force impulse (ARFI) elastography for the noninvasive diagnosis of liver fibrosis in children. Pediatr Radiol 2013; 43: 545-551

24 Goldschmidt I, Streckenbach C, Dingemann C et al. Application and limitations of transient liver elastography in children. J Pediatr Gastroenterol Nutr 2013; 57: 109-113

25 Pradhan F, Ladak F, Tracey J et al. Feasibility and reliability of the FibroScan S2 (pediatric) probe compared with the M probe for liver stiffness measurement in small adults with chronic liver disease. Ann Hepatol 2013; 12: 100-107

26 Fontanilla T, Cañas T, Macia A et al. Normal values of liver shear wave velocity in healthy children assessed by acoustic radiation force impulse imaging using a convex probe and a linear probe. Ultrasound Med Biol 2014; 40: 470-477

27 Lee MJ, Kim MJ, Han KH et al. Age-related changes in liver, kidney, and spleen stiffness in healthy children measured with acoustic radiation force impulse imaging. Eur J Radiol 2013; 82: 290-294

28 Matos $H$, Trindade A, Noruegas MJ. Acoustic radiation force impulse imaging in paediatric patients: normal liver values. J Pediatr Gastroenterol Nutr 2014; 59: 684-688

29 Hanquinet $S$, Courvoisier D, Kanavaki A et al. Acoustic radiation force impulse imaging-normal values of liver stiffness in healthy children. Pediatr Radiolo 2013; 43: 539-544

30 Engelmann G, Gebhardt C, Wenning $D$ et al. Feasibility study and control values of transient elastography in healthy children. Eur J Pediatr 2012; 171: 353-360

31 Noruegas MJ, Matos $H$, Gonçalves I et al. Acoustic radiation force impulse-imaging in the assessment of liver fibrosis in children. Pediatr Radiol 2012; 42: 201-204 